\title{
Habitats naturels des écosystèmes du Cameroun
}

\author{
Jean-Michel ONANA ${ }^{1 *}$, Jean Louis FOBANE ${ }^{2}$, Elvire Hortense BIYE ${ }^{1}$, \\ Eric Ngansop TCHATCHOUANG ${ }^{3}$ et Marguérite Marie Abada MBOLO ${ }^{1}$
}

\author{
${ }^{1}$ Université de Yaoundé I, Faculté des Sciences, Département de Biologie et Physiologie Végétales, BP. 812 Yaoundé \\ ; Herbier National du Cameroun, B.P. 1601 Yaoundé. \\ ${ }^{2}$ Université de Yaoundé I, Ecole Normale Supérieure, Département des Sciences Biologiques, B.P. 47 Yaoundé. \\ ${ }^{3}$ Institut de Recherche Agricole pour le Développement Herbier National du Cameroun, B.P. 1601 Yaoundé. \\ *Auteur correspondant ; E-mail : jeanmichelonan@gmail.com
}

\section{RÉSUMÉ}

Dans le cadre de la Stratégie et le Plan d'Action National sur la Biodiversité, afin d'améliorer la compréhension et la documentation des écosystèmes, l'objectif de cette étude est l'identification et la cartographie les habitats naturels du Cameroun. Les matériels utilisés ont été les référentiels constitués de la classification standard des habitats de l'Union Internationale pour la Conservation de la Nature (UICN) pour la nomenclature, et les cartes phytogéographique, du relief et de l'hydrographie pour les habitats terrestres et aquatiques pour les cartes thématiques respectivement. Les méthodes ont été d'adapter la classification de l'UICN à celle des phytochories pour la nomenclature ; et la fabrique des délimitations sur des fonds de cartes existantes pour la cartographie. Les résultats obtenus sont la reconnaissance de différents types d'habitats naturels du Cameroun: trois cartes qui présentent les délimitations des habitats naturels terrestres par écosystème, et aquatiques par bassin hydrographique ; et que la répartition des habitats terrestres est corrélée avec la nature des sols. Ces données complètent les connaissances sur les écosystèmes et sont un outil pour l'application de l'approche par écosystème. C'est une contribution à la cartographie des milieux naturels du Cameroun en rapport avec la gestion durable de la biodiversité.

(C) 2019 International Formulae Group. All rights reserved

Mots clés. Cameroun, habitat naturel, écosystème, phytochorie, hydrographie, cartographie.

\section{Natural habitats of the ecosystems of Cameroon}

\begin{abstract}
Within the framework of the National Biodiversity Strategy and Action Plan, to improve the understanding and documentation of the ecosystems, the objectives of this study are the identification and the mapping of natural habitats of Cameroon. The materials used have been the referentials made up of the classification standard scheme of the habitats of the International Union for the Conservation of Nature (IUCN) for nomenclature, and the phytogeographic, relief and hydrography maps for terrestrial and aquatic habitats respectively. The methods have been to adapt the IUCN classification to that of phytochoria for nomenclature; and fabricate boundaries on existing maps for cartography. The results obtain are the recognition of different natural habitat types in Cameroon: three maps showing the boundaries of terrestrial natural habitats by ecosystem, and aquatic by watershed; and that the distribution of terrestrial habitats is correlated with the
\end{abstract}


nature of the soil. These data improve the knowledge on ecosystems, and are an instrument for the implementation of the ecosystem approach. It's a contribution to the mapping of natural environments of Cameroon in relation with the sustainable management of the biodiversity.

(C) 2019 International Formulae Group. All rights reserved

Keywords: Cameroon, natural habitat, ecosystem, phytochoria, hydrography, mapping

\section{INTRODUCTION}

L'habitat naturel a été consacré en écologie ou biologie de la conservation comme unité expérimentale de base pour la recherche et l'évaluation des menaces qui pèsent sur la biodiversité. Les plantes dans ce contexte sont des organismes qui fournissent l'infrastructure par leurs composition et organisation structurelle. Dans le cadre de la stratégie mondiale pour la conservation des plantes (Secrétariat de la convention sur la biodiversité, 2002), un des axes stratégiques est de comprendre et de documenter la biodiversité dans toutes ses composantes, entre autres par l'identification, la recherche sur l'écologie et la conservation des plantes, l'amélioration de la gestion de la diversité végétale et des communautés végétales, et les habitats et écosystèmes associés, aussi bien dans des milieux naturels que dans les environnements aménagés ou habitats artificiels. En application du principe de subsidiarité, la stratégie et plan d'action national pour la biodiversité du Cameroun révisée (SPANB II) (MINEPDED, 2014) relève en son principe 6 «l'intégration de la biodiversité et des considérations écosystémiques dans tous les secteurs. Le but stratégique B est de «conserver et d'améliorer l'état de la biodiversité en préservant les écosystèmes et les habitats»; l'objectif 6 de ce principe vise à «promouvoir les plans de développement et de gestion pour toutes les zones prioritaires ou les habitats cruciaux qui sont protégés». Il apparait que les concepts d'écosystème et d'habitat sont bien distincts et que la compréhension et la connaissance des deux sont complémentaires pour la gestion durable et la conservation de la biodiversité.

C'est dans cette optique que la cartographie des écosystèmes du Cameroun et la connaissance de ses caractéristiques écologiques ont été réalisées (Onana, 2018, 2019) comme prérequis pour documenter la nomenclature et la cartographie des habitats naturels du Cameroun. Les termes souvent utilisés pour les aires écologiques à l'intérieur des écosystèmes sont variés et parfois imprécis. Ainsi il est question d'espace végétalisé urbain (Charahabil et al., 2018), de milieux salé et acide (Thiam et al., 2015), de zone soudanienne (Ouattara et al., 2016), ou même d'écosystèmes (Folahan et al., 2018), parfois des termes empruntés dans d'autres disciplines tel que la géomorphologie en parlant de hautes terres (Tiokeng et al., 2015) ou à l'hydrographie pour le bassin versant (Garba, 2017), toutes choses qui créent une confusion dans la terminologie des divisions de l'écosystème. Par contre pour l'écologie et la biologie de la conservation, il est clair que pour l'évaluation des menaces qui pèsent sur les espèces ou les milieux de vie d'organismes, la division de l'écosystème est l'habitat (Limoges et al., 2013 ; IUCN, 2016, 2018a). C'est pourquoi l'UICN a entrepris de proposer une classification standard des habitats (IUCN, 2018b).

En sciences naturelles, le terme habitat est un concept d'abord utilisé dans le domaine de l'écologie (Sadik, 2013). Un habitat se définit alors par une biocénose et le milieu physique qui l'héberge. C'est ainsi l'environnement immédiat de l'organisme vivant caractérisé par les facteurs physiques (Beentje, 2010). Selon plusieurs auteurs, le terme habitat est synonyme de biotope qui est le lieu de vie défini par des caractéristiques physiques et chimiques relativement stables. Cependant, alors que le biotope héberge un ensemble de formes de vie composant la biocénose constituée de la flore, la faune, les champignons et des populations de microorganismes, l'habitat concerne seulement une espèce ou une (sous) population d'une espèce 
(UICN, 2012). En cela, les habitats ont théoriquement leurs caractéristiques propres à l'intérieur de l'écosystème. Certaines espèces jouent d'ailleurs un rôle essentiel dans la création et l'entretien de leur propre habitat (cas des coraux pour les récifs coralliens ou des termites pour les termitières).

En biologie ou écologie de la conservation, discipline traitant des questions de perte, maintien ou restauration de la biodiversité et qui vise à comprendre les causes de la perte de biodiversité, à l'enrayer et à promouvoir l'utilisation durable des ressources naturelles (Onana, 2019), l'habitat est le lieu, le type de site ou d'endroit, l'environnement naturel dans lequel un organisme vit et grandit ou une population existe à l'état naturel (Secrétariat de la Convention sur la diversité biologique, 2004). Le terme se réfère donc typiquement à un espace particulier dans lequel un organisme vit et où il peut trouver des éléments nutritifs, un abri, une protection et des conditions de fécondation pour se reproduire. Au regard d'une espèce, il peut s'agir: d'un logement à proprement parler (un nichoir, un terrier) ; d'un espace vital pour une espèce (ensemble d'éléments du paysage qui offrent les ressources suffisantes pour la survie et la reproduction d'une population ou d'une espèce) ; du biotope (lieu de vie avec des caractéristiques physiques, chimiques, climatiques, géomorphologiques, édaphiques relativement stables); d'une aire avec des conditions environnementales uniformes associées à un ensemble de formes de vie formant une communauté particulière qu'elle héberge composant la biocénose; de la niche écologique (ensemble de conditions environnementales telles qu'une espèce donnée peut former des populations viables lorsqu'il s'agit de décrire un besoin particulier de l'espèce, chaque espèce étant identifiée par des seuils écologiques qu'elle admet vis-à-vis de chaque paramètre et qui dessinent les contours de la niche). A cause de la dimension et de son caractère parfois variable, la niche écologique peut être tellement réduite qu'on parle de micro-habitat (on distingue le microhabitat optimal dans lequel l'individu trouve l'optimum des conditions environnementales pour sa survie et sa reproduction, et le microhabitat sous-optimal qui est un milieu de substitution naturel ou artificiel) où l'espèce survit, mais difficilement et avec un succès reproductif anormalement bas (UDEV, 2013). L'habitat peut aussi être un site défini comme une aire géographique terrestre et/ou aquatique avec des frontières écologiques, physiques, administratives ou d'aménagement qui est actuellement ou potentiellement peutêtre aménagée comme une unité singulière (IUCN, 2016; Darbyshire et al., 2017); ou une localité uniquement s'il y a une menace identifiée dans le cadre de l'évaluation des menaces d'extinction qui pèsent sur une espèce (UICN, 2012). On distingue différents types d'habitat :

- l'habitat naturel : unité naturelle, bien identifiable, essentiellement caractérisée par sa végétation, son climat, son exposition, son altitude, son sous-sol et son sol;

- l'habitat semi-naturel : milieu qui réunit les conditions physiques et biologiques nécessaires à l'existence d'une espèce ou d'un groupe d'espèces animales ou végétales et caractérisé par les activités humaines qui s'y déroulent;

- l'habitat artificiel : habitat de substitution ou construit artificiellement.

Le présent travail s'intéresse essentiellement aux habitats naturels bien que la signification du qualificatif "naturel" fasse aujourd'hui l'objet de questionnement par certains biogéographes contemporains (Gillson et al., 2011). Un habitat naturel est compris comme celui qui n'a pas subi de dégradation due aux activités humaines de nature industrielle ou tout au moins de grande ampleur. Toutefois il sera évoqué, au moins dans la nomenclature et subsidiairement sur la carte, les habitats semi-naturels et artificiels car ils résultent de la dégradation (modification), de la déforestation (conversion) (Onana, 2019; Tchatchou et al. 2015) de l'habitat naturel ou d'une construction dans l'habitat naturel (cas des retenues d'eau).

La notion d'habitat ayant une compréhension large telle que présentée cidessus, il a été nécessaire d'établir une nomenclature et une classification. La 
classification standard de l'UICN (2018b) défini les types d'habitats majeurs à trois niveaux dont huit du niveau 1: forêt, savane, formation arbustive, formation herbeuse, milieu humide, milieu rocheux, cave, et désert. Chaque type d'habitat majeur de niveau 1 est subdivisé en habitats majeurs de niveau 2. Le niveau 3 n'existe que pour les habitats des récifs coralliens et de la Mer entre 200 et $4000 \mathrm{~m}$ de profondeur. Etant donné que cette classification est un document de travail, elle est adaptée au Cameroun ainsi qu'il suit:

1) habitat majeur (niveau 1) assimilable à l'écosystème, au biome, à la biorégion, à la région phytogéographique ou au centre d'endémisme d'espèces végétales;

2) habitat principal (habitat majeur de niveau 2) assimilable aux domaines, secteurs phytogéographiques ou sous-centre d'endémisme d'espèces végétales;

3) habitat sensu stricto assimilable au district phytogéographique ou au groupement végétal;

4) site comme division de l'habitat.

Le concept d'habitat est aussi utilisé pour décrire et éventuellement cartographier l'endroit, et les caractéristiques du milieu comme étant celui dans lequel une population d'individus d'une espèce donnée (ou d'un groupe d'espèces symbiotes ou vivant en guilde) peuvent normalement vivre et s'épanouir. Pour mieux connaître et protéger la biodiversité, et évaluer les impacts des activités humaines et du dérèglement climatique sur cette biodiversité, le besoin est apparu de cartographier de manière assez précise les habitats d'espèces. La fonction de cartographie permet alors de représenter les unités écologiques fonctionnelles à l'intérieur desquels s'opèrent les processus naturels. Selon le Secrétariat de la Convention sur la diversité biologique (2004) dans l'approche par écosystème, la définition de l'habitat mentionne l'unité ou l'échelle spatiale de l'organisme ou de la population soumise à l'aménagement. Aussi la taille d'une aire protégée influence les mesures d'aménagement à mettre en œuvre pour la conservation (Gillson et al., 2011; Ridle et al., 2011). La cartographie des habitats est alors justifiée en ce qu'elle permet de visualiser les délimitations des unités écologiques. C'est dans cette optique que la cartographie des écosystèmes du Cameroun avait été réalisée avec la perspective de compléter la documentation par celle des habitats (Onana, 2018). Car pour la conservation, la connaissance de l'habitat est essentielle afin d'évaluer les menaces d'extinction qui pèsent sur un taxon ou le choix d'espèces pour la restauration des sites perturbés (UICN, 2010b). Le critère de rang $B$ de la répartition géographique consacre ainsi les aires d'occupation et d'occurrence comme données à utiliser pour l'étendue et l'état de santé environnementale de l'habitat. C'est d'ailleurs le critère le plus utilisé parce que les données sont généralement disponibles, et on dispose d'outils de mesure et d'analyse (Bachman et Moat, 2012; Bachman et al., 2011; Willis et al., 2003). Et cela est confirmé par différents travaux d'évaluation de l'état de menace d'extinction qui pèse sur les espèces à cause du déclin continue ou de la réduction de la superficie, étendue et /ou qualité de l'habitat (IUCN, 2014a, 2014b; Schatz et al., 2019), et aussi dans environ 66\% des 461 espèces végétales du Cameroun (environ $70 \%$ en Australie, $62 \%$ globalement); ce chiffre monte à $92 \%$ pour les espèces végétales endémiques pour le Cameroun (75\% pour l'Australie) (IUCN, 2017; Le Breton et al., 2019 ; République du Cameroun, 2014; Onana, 2013; Onana et Cheek, 2011), et environ $75 \%$ des 236 espèces animales menacées d'extinction du Cameroun (IUCN, 2017).

L'objectif de ce travail est de proposer une nomenclature et une cartographie des habitats naturels qu'on trouve sur le territoire du Cameroun. La cartographie des habitats vise à compléter celle des écosystèmes afin de disposer d'outils géographiques de la répartition des milieux naturels du Cameroun selon les standards du développement durable.

En réalisant la cartographie des habitats naturels après celle des écosystèmes, le présent travail sert aussi à représenter en même temps les concepts d'écosystèmes et $\mathrm{d}$ 'habitat dans une configuration spatiale qui clarifient l'étendue des unités d'un concept par rapport à l'autre et ainsi l'utilisation moins confuse de chacun des termes grâce à la 
visualisation des cartes: les écosystèmes sont de l'étendue des régions phytogéographiques tandis que les habitats sont de celle des divisions inférieures que sont les domaines, secteurs, districts, ou à une échelle plus petite des localités et sites. Aussi pour les besoins de la conservation des espèces ou des écosystèmes, les données sur l'habitat sont essentiels, étant plus accessibles et (Moat et Bachman, 2012 ; Le Breton et al., 2019).

L'intérêt du travail est de donner des arguments scientifiques pour l'application plus rigoureuse de la description des milieux de vie des organismes vivants et aussi des critères d'évaluation du degré de menace qui pèse sur ces organismes en relation avec l'état de santé de leur environnement, afin d'établir leur statut de conservation. Ce dernier est en effet un outil d'aide à la décision pour la planification du développement, la gestion durable et la conservation de la biodiversité. C'est une contribution de la recherche aux objectifs pour la compréhension et la documentation de la biodiversité spécifique aux écosystèmes (MINEPDED, 2014).

\section{MATERIEL ET METHODES Matériel}

Le référentiel pour la nomenclature des habitats est la classification standard de l'UICN (2012) qui présente les habitats majeurs à trois niveaux: niveau 1 , niveau 2 et niveau 3 des récifs coralliens et de la plaine abyssale (IUCN, 2018a). C'est un document de travail où il est reconnu que cette classification n'est pas entièrement satisfaisante car plusieurs types d'habitats restent à définir. Cependant il est le référentiel pour les biologistes de la conservation. Au Cameroun, Letouzey (1985) a rigoureusement décrit les unités phytogéographiques correspondant aux deux premiers niveaux de classification, mais aussi va plus loin en décrivant les groupements végétaux des sites ; de même que Tchawa (2006) reste jusqu'alors la meilleure cartographie du relief et des principaux cours d'eau. Ce sont donc des bases robustes pour déterminer et cartographier les habitats naturels terrestres et aquatiques des écosystèmes du Cameroun.

\section{Méthodes}

Comme pour les écosystèmes, la méthode consiste à exploiter les référentiels bibliographiques déjà publiées (Witté et Touroult, 2014; Onana, 2018). En effet selon Gros-Desormeaux (2015), la représentation graphique d'unités d'intérêt écologique, faunique ou floristique a plutôt tendance à mobiliser des représentations qui s'appuient sur des informations géographiques préalablement traduites en cartes, car ils permettent l'agrégation des données géographiques. Pour cartographier les habitats, c'est la méthode de fabrique des délimitations sur des fonds de carte déjà connues et choisi en fonction des paramètres retenus qui a été utilisée. Elle consiste en la construction des tracés qui délimitent les unités cartographiques par une approche cognitive permettant de dessiner sur un fond de carte un contour jugé pertinent. Les tracés pour les habitats terrestres se faits sur fond de la carte phytogéographique du Cameroun où les habitats sont délimités en fonction des groupements végétaux, y compris les formations particulières occupant de petites superficies mais présentant des caractéristiques écologiques différentes; et pour les habitats aquatiques, c'est la carte du relief et hydrographie où la construction des tracés délimite les bassins versants.

\section{RESULTATS}

Ils portent sur la nomenclature et la cartographie des principaux habitats terrestres et aquatiques. Il est fait mention aussi d'habitats semi-naturels et artificiels pour la nomenclature, mais ces derniers ne sont pas cartographiés.

\section{Nomenclature des habitats}

La correspondance entre les classes standards d'habitats de l'UICN (IUCN, 2018b) et les principaux habitats du territoire du Cameroun est présentée dans le Tableau 1. Les principaux habitats naturels du Cameroun par écosystème sont présentés dans le Tableau 2.

Les légendes des habitats qui accompagnent les cartes donnent la désignation des habitats selon les cartes. 


\section{Cartographie des habitats}

Les cartes des habitats présentent la distribution spatiale et les délimitations des habitats. La carte des principaux habitats (Figure 1) montre ainsi des unités cartographiques et la carte des habitats terrestres (Figure 2) montre les habitats sensu stricto par unité. La légende qui accompagne cette dernière donne la composition floristique synoptique avec indication d'espèces caractéristiques des groupements végétaux dominants de chaque habitat. Ces groupements végétaux varient aussi à l'intérieur des habitats dans les sites. Certains sites de très petite superficie, à cause de la particularité qu'ils impriment, sont signalés sur la carte (cas d'affleurements de rocher ou de cuirasse latéritique pour les espèces saxicoles des habitats terrestres dans l'Adamaoua).

A l'observation, il y a une corrélation entre les types de sols et les types d'habitat (Tableau 3). La nature du sol en effet conditionne le type de végétation qui s'y développe (cas des halophytes qui se développent sur les sols salées et qui occupent souvent de petites superficies dans les écosystèmes semi-arides (Thiam et al., 2016 ; Laban et al., 2018). Ainsi pour la plupart des habitats, les types de sol sont spécifiques, bien que parfois certains types se trouvent dans plusieurs habitats d'un même écosystème. Les sols sont plus variés avec souvent juxtaposition dans les habitats de l'écosystème semi-aride, tandis qu'ils semblent plus homogènes dans les écosystèmes de forêt tropicale dense humide et de montagne, d'où des habitats qui forment des blocs sur de grandes superficies.

La carte des habitats aquatiques (Figure 3) sur fond de carte du relief montre la répartition des habitats par bassin hydrographique (ou bassin versant), car ceuxci dépendent étroitement de ce dernier, des régimes hydrographiques et hydrologiques locaux. Il n'a pas été indiqué des habitats de très petites superficies tels que les affleurements immergés pour les plantes saxicoles, ou des sites de chutes d'eau et de rapides qui sont des habitats spécifiques pour les espèces saxicoles immergées tel que les Podostemaceae.

Tableau 1 : Correspondance de la classification standard des habitats de l'UICN et des principaux habitats au Cameroun (la numérotation des habitats suit la codification globale de la classification standard de l'UICN).

\begin{tabular}{ll}
\hline Classification standard de l'UICN & Habitat correspondant au Cameroun \\
\hline $\begin{array}{l}\text { A- Habitats naturels } \\
\text { Habitats majeurs }\end{array}$ & Principaux habitats \\
\hline $\begin{array}{l}\text { 1. Forêt } \\
\text { 1.6 Forêt tropicale humide }\end{array}$ & $\begin{array}{c}\text { Forêts tropicales denses humides toujours vertes ou } \\
\text { semi-caducifoliées (ou semi-décidues) de basse et } \\
\text { moyenne altitude, forêts mixtes, forêts rupicoles. }\end{array}$ \\
$\begin{array}{l}\text { 1.7 Forêt tropicale de végétation de } \\
\text { mangrove au-dessus des marées } \\
\text { hautes }\end{array}$ & \begin{tabular}{l} 
Mangroves. \\
\hline
\end{tabular} \\
\hline
\end{tabular}

2. Savane

2.1 Savane sèche

2.2 Savane humide
Steppes à épineux, savanes sahélo-soudaniennes savanes soudano- sahéliennes.

Savanes soudaniennes, savanes médio-soudaniennes, savanes soudano-guinéennes, savanes guinéosoudaniennes ou périforestières.

\section{Boisement arbustif}

3.5 Boisement arbustif tropical sec

Boisements arbustifs sahélo-soudaniens. 


\begin{tabular}{|c|c|}
\hline $\begin{array}{l}\text { 4. Prairie } \\
\text { 4.6 Prairie tropicale périodiquement } \\
\text { inondée de basse altitude } \\
\text { 4.7 Prairie tropicale de haute altitude }\end{array}$ & $\begin{array}{l}\text { Formations végétales de zones humides } \\
\text { périodiquement inondables, prairies marécageuses. } \\
\text { Prairies d'altitude, prairies subalpines. }\end{array}$ \\
\hline \multicolumn{2}{|l|}{ 5. Zones humides (eau douce) } \\
\hline $\begin{array}{l}5.1 \text { Rivières, ruisseaux, chute d'eau } \\
\text { permanente }\end{array}$ & Fleuves, rivières, chutes d'eau. \\
\hline $\begin{array}{l}5.2 \text { Rivières, ruisseaux, chute d'eau } \\
\text { périodique }\end{array}$ & Fleuves, rivières. \\
\hline $\begin{array}{l}\text { 5.4 Marécages, marais, tourbières } \\
\text { (généralement plus de } 8 \text { ha) }\end{array}$ & Formations marécageuses, tourbières. \\
\hline $\begin{array}{l}5.5 \text { Lac d'eau douce permanent (plus } \\
\text { de } 8 \text { ha) }\end{array}$ & Lac. \\
\hline $\begin{array}{l}\text { 5.6 Lac saisonnier/ intermittent (plus } \\
\text { de } 8 \text { ha), y compris les zones } \\
\text { inondables des plaines }\end{array}$ & Zones inondables: zones périodiquement inondables. \\
\hline $\begin{array}{l}\text { 6. Zones rocheuses (ex. escarpement, } \\
\text { falaise, rocher immergé, sommet de } \\
\text { montagne) }\end{array}$ & $\begin{array}{l}\text { Groupements saxicoles, affleurements rocheux, } \\
\text { cuirasse latéritique, endroits des chutes d'eau rapides } \\
\text { des cours d'eau. }\end{array}$ \\
\hline \multicolumn{2}{|l|}{ 9. Zone marine [néritique] } \\
\hline 9.1 Littoral [pélagique] & Forêt tropicale dense humide littorale. \\
\hline 9.4 Plage sableuse & Forêts sur cordons littoraux. \\
\hline 9.10 Estuaire & Mangroves, forêts marécageuse d'arrière mangrove. \\
\hline \multicolumn{2}{|l|}{ 10. Marin aquatique } \\
\hline $\begin{array}{l}\text { 10.1 Plateau continental } \\
\text { [Epipélagique] }(0-200 \mathrm{~m})\end{array}$ & Plateau continental. \\
\hline \multicolumn{2}{|l|}{ 12. Marin [intertidal] } \\
\hline 12.7 Mangrove à racines immergées & Mangrove. \\
\hline \multicolumn{2}{|l|}{ B- Habitats semi naturels ou artificiels } \\
\hline \multicolumn{2}{|l|}{ 14. Artificiel-Terrestre } \\
\hline 14.1 Terre arable & Plantations, cultures, fermes agricoles. \\
\hline 14.2 Pâturages & Fermes agricoles, faciès de savane dégradée. \\
\hline 14.3 Plantations & Forêts plantées, vergers, champs (cultures vivrières). \\
\hline 14.4 Jardins ruraux & $\begin{array}{l}\text { Jardins de case, cultures/élevage d'exploitation } \\
\text { familiale, jachères. }\end{array}$ \\
\hline 14.5 Zones urbaines & Jardins urbains, broussailles. \\
\hline $\begin{array}{l}\text { 14.6 Ancienne forêt tropicale } \\
\text { fortement dégradée }\end{array}$ & Forêts secondaires, broussailles. \\
\hline \multicolumn{2}{|l|}{ 15. Artificiel-aquatique } \\
\hline 15.1 Retenues d'eau (plus de 8 ha) & \\
\hline 15.2 Etang, mare & Etangs piscicoles, mares d'eau. \\
\hline 15.3 Etang piscicole & Etangs piscicoles. \\
\hline 15.6 Zone d'épuration des eaux & Zones d'épuration des eaux. \\
\hline 16. Végétation introduite & $\begin{array}{l}\text { Jardins botaniques, jardins de case, plantes rudérales, } \\
\text { broussailles d'espèces envahissantes. }\end{array}$ \\
\hline
\end{tabular}


Tableau 2 : Principaux habitats terrestres des écosystèmes du Cameroun

\begin{tabular}{|c|c|c|}
\hline Ecosystème & Phytochories & Principaux habitats \\
\hline \multirow[t]{2}{*}{$\begin{array}{l}\text { 1. Eau douce (eaux peu } \\
\text { ou pas courantes) }\end{array}$} & $\begin{array}{l}\text { Région soudanienne, domaine sahélien, - } \\
\text { secteur sahélien }\end{array}$ & $\begin{array}{l}\text { Prairies inondables sahélo- } \\
\text { soudaniennes de la vallée du } \\
\text { Logone. }\end{array}$ \\
\hline & $\begin{array}{l}\text { Région guinéo-congolaise; domaine des } \\
\text { forêts tropicales denses humides toujours } \\
\text { vertes guinéo-congolaises, - secteur } \\
\text { forestier toujours vert camerouno- } \\
\text { congolais ou congolais du Dja }\end{array}$ & $\begin{array}{l}\text { Forêt marécageuse du Haut- } \\
\text { Nyong; raphiales. }\end{array}$ \\
\hline 2. Semi-aride & $\begin{array}{l}\text { Région soudanienne, domaine sahélien, - } \\
\text { secteur sahélien }\end{array}$ & $\begin{array}{l}\text { Steppes arbustives à } \\
\text { épineux; boisements sahélo- } \\
\text { soudaniens à épineux encore } \\
\text { dominants. }\end{array}$ \\
\hline \multirow[t]{5}{*}{$\begin{array}{l}\text { 3. Savane tropicale } \\
\text { boisée }\end{array}$} & $\begin{array}{l}\text { Région soudanienne, domaine soudanien } \\
\text { - secteur soudano-sahélien }\end{array}$ & $\begin{array}{l}\text { Savanes arborées et } \\
\text { arbustives sahélo- } \\
\text { soudaniennes; savanes } \\
\text { boisées soudano- } \\
\text { sahéliennes. }\end{array}$ \\
\hline & - secteur médio-soudanien & $\begin{array}{l}\text { Savanes boisées médio- } \\
\text { soudaniennes. }\end{array}$ \\
\hline & - secteur soudano-guinéen d'altitude & $\begin{array}{l}\text { Savanes arbustives } \\
\text { soudano-guinéennes } \\
\text { d'altitude; savanes boisées } \\
\text { soudano-guinéennes } \\
\text { d'altitude, forêts claires. }\end{array}$ \\
\hline & $\begin{array}{l}\text { Région guinéo-congolaise, étage de basse } \\
\text { et moyenne altitude, domaine des forêts } \\
\text { denses humides semi-décidues guinéo- } \\
\text { congolaises }\end{array}$ & \\
\hline & $\begin{array}{l}\text { - secteur des savanes guinéo-congolaises } \\
\text { ou péri-forestières parfois } \\
\text { submontagnardes }\end{array}$ & $\begin{array}{l}\text { Savanes guinéo- } \\
\text { soudaniennes (ou péri- } \\
\text { forestière) à Terminalia } \\
\text { macroptera dans le plateau } \\
\text { de Adamaoua, à } T \text {. mollis } \\
\text { sur le plateau central et la } \\
\text { plaine de l'est et à } T \text {. } \\
\text { glaucescens sur les hautes } \\
\text { terres de l'ouest. }\end{array}$ \\
\hline \multirow[t]{2}{*}{ 4. Montagne } & $\begin{array}{l}\text { Région soudanienne, domaine sahélien, - } \\
\text { secteur soudano-sahélien d'altitude }\end{array}$ & $\begin{array}{l}\text { Savanes boisées médio- } \\
\text { soudaniennes d'altitude sur } \\
\text { les Monts Mandara. }\end{array}$ \\
\hline & $\begin{array}{l}\text { Région afromontagnarde } \\
\text { - étage afro-subalpin } \\
\text { - étage montagnard }\end{array}$ & $\begin{array}{l}\text { Prairies subalpines. } \\
\text { Prairies montagnardes; }\end{array}$ \\
\hline
\end{tabular}


- étage submontagnard forêts montagnardes.

Forêts submontagnardes; savanes arbustives

d'altitude.

\begin{tabular}{|c|c|c|}
\hline $\begin{array}{l}\text { 5. Forêt tropicale dense } \\
\text { humide }\end{array}$ & $\begin{array}{l}\text { Région guinéo-congolaise, étage de } \\
\text { basse et moyenne altitude, domaine des } \\
\text { forêts tropicales denses humides } \\
\text { - secteur forestier semi-caducifolié (ou } \\
\text { semi-décidu) sensu stricto }\end{array}$ & $\begin{array}{l}\text { Forêts semi-décidues ; } \\
\text { forêts mixtes à } \\
\text { prépondérance d'éléments } \\
\text { semi-décidus. } \\
\text { Forêts atlantiques de type } \\
\text { nord-occidental; forêts } \\
\text { atlantiques biafréennes; } \\
\text { forêts atlantiques littorales, } \\
\text { forêts atlantiques centrales } \\
\text { et orientales; forêts mixtes à } \\
\text { prépondérance d'éléments } \\
\text { toujours verts atlantiques. } \\
\text { Forêts congolaises du Dja ; } \\
\text { forêts mixtes à } \\
\text { prépondérance d'éléments } \\
\text { toujours verts congolaises } \\
\text { du Dja. }\end{array}$ \\
\hline 6. Marin et côtier & $\begin{array}{l}\text { Région guinéo-congolaise, étage de basse } \\
\text { et moyenne altitude, domaine } \\
\text { des forêts tropicales denses humides } \\
\text { toujours vertes guinéo-congolaises } \\
\text { - secteur forestier toujours vert atlantique } \\
\text { ou nigéro-camerouno-gabonais }\end{array}$ & $\begin{array}{l}\text { Mangroves; forêts sur } \\
\text { cordons littoraux; forêts } \\
\text { marécageuse d'arrière } \\
\text { mangrove. }\end{array}$ \\
\hline
\end{tabular}




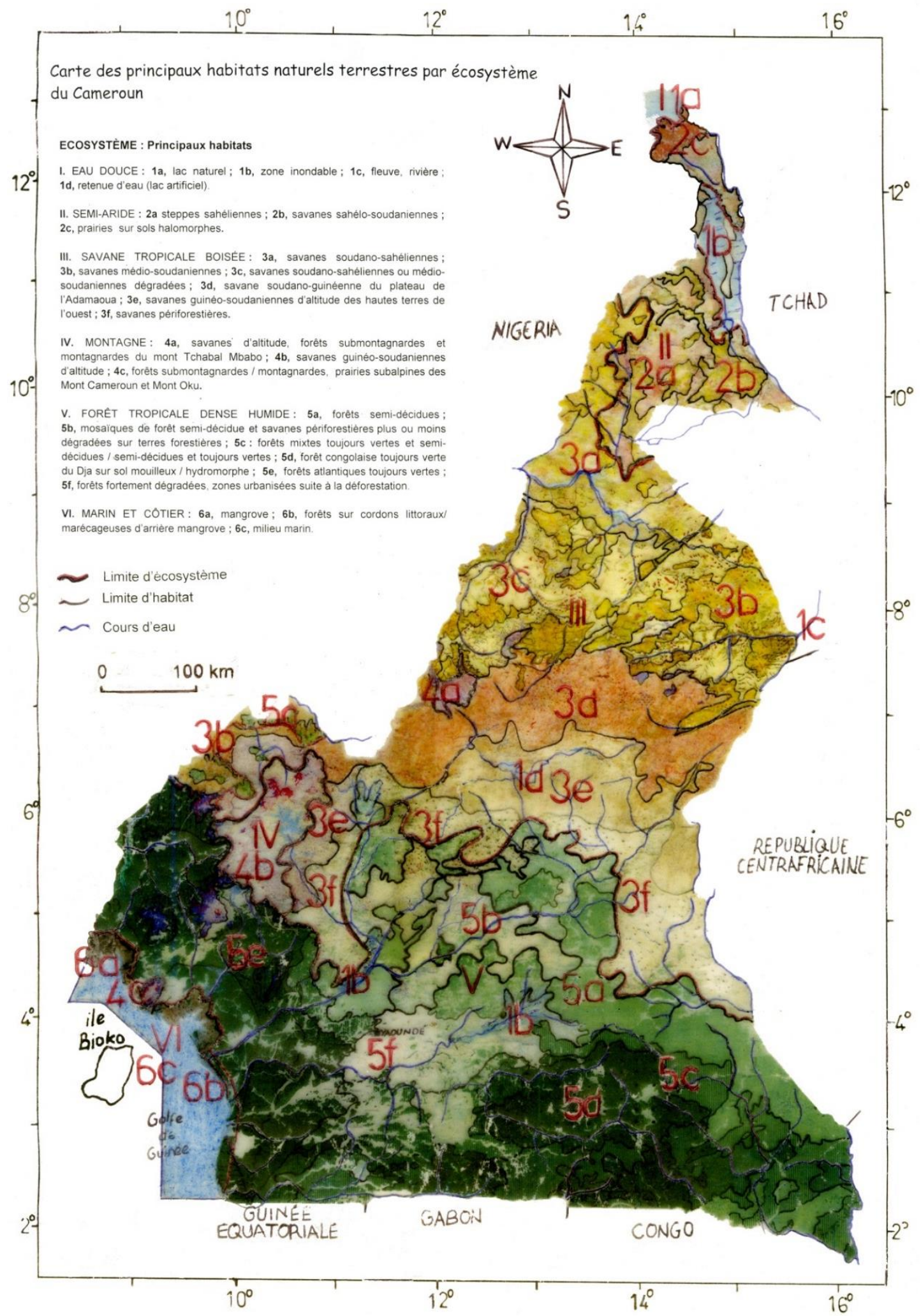

Figure 1 : Carte des principaux habitats naturels terrestres du Cameroun basée sur la carte phytogéographique (Letouzey, 1985). 


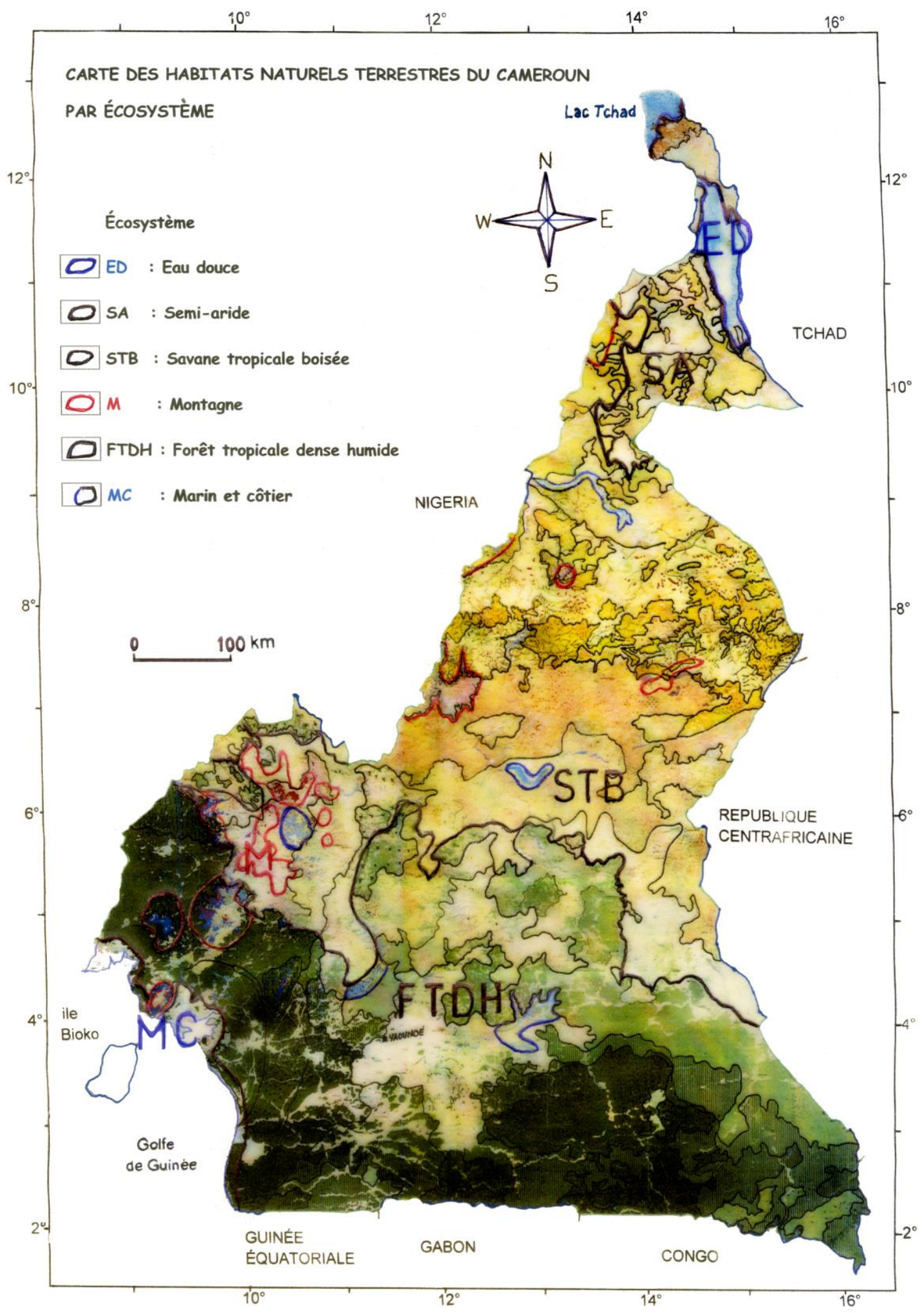

Figure 2 : Carte des habitats naturels terrestres du Cameroun par écosystème basée sur la carte phytogographique (Letouzey, 1985). 


\section{Légende de la carte des habitats naturels terrestres du Cameroun par écosystème}

Ecosystème d'eau douce

[1] Iles flottantes et eaux libres du Lac Tchad

Prairies inondables (Yaérés) graminéennes

Forêts marécageuses du Nyong à Sterculia subviolacea et Macaranga assas

Dépressions marécageuses périodiquement inondées du confluent Mbam-Sanaga à Loudetiospis ambiens et Vetiveria nigritana avec ceinture de Borassus aetiopicum

Savanes de Dépressions marécageuses périodiquement inondées du confluent Mbam-Sanaga à Zanthoxylum zanthozyloides avec avancée d'éléments soudani ns en particulier Lo hira lanceolato

[1] Forêts marécageuses périodiquement inondées de basse et moyenne altitude, parfois en taches circulaires (Plaine des Mbos près de Nkongsamba)

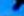

Forêts marécageuses périodiquement inondées d'altitude (>1000m), plaine de Ndop

Eaux libres (fleuves, lacs, rivières)

Ecosystème semi-aride

Steppes à épineux (Ziziphus mauritiana, Acacia spp, Balanites aegyptiaca) sahéliennes et boisements

(4) Jachères et steppes arborees et arbustives sahélosoudaniennes dégradées

C.) Steppes arbustives sahélo-soudaniennes et soudanoSahéliennes

Ecosystème de savane tropicale boisée Savanes arbustive et arborée soudano-sahéliennes et boisements

Savane boisée médio-soudaniennes de moyenne altitude $( \pm 600-900 \mathrm{~m})$ à Isoberbilina doka et boisements sur les Monts Mandara

Savanes boisées médio-soudaniennes à Isoberlinia doka , Afzelia africana et Anogeissus leiocarpus ; peuplements \pm denses de Monotes kerstingii, Parinari curatifolia et Uapaca togoensis parfois dégradées

Jachères et savanes boisées médio-soudaniennes dégradées parfois pâturées sur sol filtrant de pente caillouteuse ou d'arènes granitiques; parfois sur cuirasses plus ou moins affleurantes; parfois avec tâche de sol hydromorphe

13 Savanes arbustives et arborées voire boisées soudanoguinéennes à Daniella oliveri et Lophira lanceolata du plateau de I'Adamaoua (alt. 800-1200 m), faciès et savanes arbustives et arborées soudano-guinéennes à Lophira lanceolata, Terminalia laxiflora, T. mollis, T. macroptera, Samanea eriorachis, voire boisées à Burkea africana, parfois dégradées par le pâturage permanent ou des cultures

3.2. Végétation soudano-guinéenne de collines chaotiques du plateau de l'Adamaoua à Hymenodyctyon floribundum

Végétation soudano-guinéenne du plateau de l'Adamaoua sur cuirasses ferrugineuses \pm démantelées à Ctenium newtonii; parfois sur dalles gréseuses (Bagodo, Meiganga)

7.9 Savanes périforestières arbustives à Terminalia glaucescens parfois pâturées jusque vers 1000-1200 m ; savanes intra- et péri-forestières chétivement arbustives à Annona senegalensis et Bridelia ferruginea

Savanes périforestières arbustives ou de transition à Terminalia glaucescens

$\because \because \bigcirc$ Mosaïques de savanes périforestières arbustives à Terminalia glaucescens et/ou taches ou éléments de forêt semi-caducifoliée
12 Savanes péri forestières avec indices soudano-sahéliens (Akwaya, Nkambé) ; faciès à Prosopis africana et Terminalia leiocarpo (Nkambé), Terminalia leiocarpa (Mayo Darlé)

Cis. Savanes arbustives et arborées voire boisées soudano-guinéennes avec "taillis arbustif d'origine pastorale"

Groupements saxicoles divers

Ecosystème de montagne

Prairies subalpines (Mt cameroun 2800-3000 à 3600-3800 m d'altitude ; Mt Oku 2800-3011m d'altitude

Forêts montagnardes (1800-2000 à 3000m)

Forêts submontagnardes (800-1200 à 1800-2200 m)

$[\because$ Pâturages montagnards (savanes herbeuse d'altitude) à Sporobolus africanus; galeries forestières

Fcosystème de forêt tropicale dense humide

cos Forêt semi-caducifoliée de type septentrional à Sterculiaceae et Ulmaceae

Forêts semi-caducifoliées à Sterculiaceae et Ulmaceae

1. Faciès de dégradation très prononcé de forêt semi-caducifoliées

Recrus forestiers de type semi-caducifolié avec éventuellment des îlots forestiers

11 Forêts mixtes semi-caducifoliée et atlantique toujours verte ou semi-caducifoliées et congolaise du Dja avec prédominance d'éléments de forêt semi-caducifoliée

Forêts atlantiques à Caesalpinioideae rares de type nordOccidental

act Forêts atlantiques à Caesalpinioideae rares de type nordoccidental avec avancée d'éléments semi- caducifoliés (zone de Ndikiniméki)

Forêts atlantiques biafréennes à Caesalpinioideae grégraires abondantes

Forêts atlantiques biafréennes à Caesalpinioideae encore abondantes avec Saccoglostis gabonensis et avancée d'autres éléments littoraux

Forêts atlantiques biafréennes à Caesalpinioideae encore abondantes avec Calpocalyx hetzii et Saccoglostis gabonensis (zone de Campo) Forêts atlantiques littorales à Lophira alata et Saccoglostis gabonensis

Forêts mixtes atlantique toujours verte ou congolaise du $\mathrm{Dj}$ toujours verte et semi-caducifoliée avec prédominance d'éléments toujours verts

Forêts atlantiques à Ceasalpinioideae rares de type central et oriental

5. Forêts congolaise du Dja avec avancées d'elements atlantiques biafréens (Coula edulis)

Forêts toujours vertes du Dja sur sol mouilleux (sol hydromorphe) avc Uapaca paludosa et autres $U$. spp. dans les vallées Eléments de forêt semi-caducifoliée dans la forêt biafréenne

Ecosystème marin et côtier

Mangroves basses à petits Rhizophora racemosa et Pandanus satablei et hautes à grands Rhizophora racemosa et Pandanus candelabrum

Lile Forêts sur cordons littoraux avec Saccoglostis gabonensis et Klainedoxa microphylla sur sable et Anthostema aubryanum et Ctenolophon englerianus sur vase

Zones totalement dégradées dans les écosystèmes

Agglomérations, couverture végétale absente, cultures, plantations 
Tableau 3 : Corrélation entre habitats et types de sols qui montre le caractère déterminant de la nature du sol sur le type d'habitat.

\begin{tabular}{|c|c|c|}
\hline Ecosystème & Habitat principal & Nature des sols \\
\hline \multirow[t]{3}{*}{ Eau douce } & $\begin{array}{l}\text { Formations } \\
\text { marécageuses }\end{array}$ & $\begin{array}{l}\text { Associations de sols ferralitiques jaunes } \\
\text { et sols hydromorphes indifférenciés. }\end{array}$ \\
\hline & Prairie inondable & $\begin{array}{l}\text { Sols hydromorphes, à gley vertiques } \\
\text { ("yaérés") de la vallée du Logone. }\end{array}$ \\
\hline & Forêt inondable & $\begin{array}{l}\text { Sols hydromorphes, humides à gley, à } \\
\text { anmoor acide du Haut Nyong. }\end{array}$ \\
\hline \multirow[t]{3}{*}{ Semi-aride } & Steppe à épineux & Sols minéraux bruts peu évolués. \\
\hline & Steppe arborée ou arbustive & Vertisols topomorphes. \\
\hline & Savane sahélo-soudanienne & Planosols et sols solonetziques. \\
\hline \multirow[t]{6}{*}{$\begin{array}{l}\text { Savane tropicale } \\
\text { boisée }\end{array}$} & Savane soudano-sahélienne & $\begin{array}{l}\text { Vertisols topomorphes et lithomorphes, } \\
\text { fersialitiques rouges et bruns, les deux en } \\
\text { associations, bruns eutrophes tropicaux. }\end{array}$ \\
\hline & Savane soudanienne & $\begin{array}{l}\text { Sols ferrugineux lessivés, hydromorphes, } \\
\text { lessivés planosoliques sur socle. }\end{array}$ \\
\hline & Savane médio-soudanienne & $\begin{array}{l}\text { Sols ferrugineux à concrétions et } \\
\text { cuirasses, ferrugineux érodés, indurés, } \\
\text { ferrugineux peu lessivés. }\end{array}$ \\
\hline & $\begin{array}{l}\text { Savane soudano-guinéenne } \\
\text { de l'Adamaoua }\end{array}$ & $\begin{array}{l}\text { Sols ferralitiques rouges, alliatiques } \\
\text { nodaux, orthiques rajeunis et appauvris. }\end{array}$ \\
\hline & $\begin{array}{l}\text { Savane guinéo-soudanienne } \\
\text { (ou péri-forestière) des hautes } \\
\text { terres de l'ouest }\end{array}$ & $\begin{array}{l}\text { Sols ferralitiques orthiques remaniés, } \\
\text { ferrugineux peu lessivés. }\end{array}$ \\
\hline & $\begin{array}{l}\text { Savane guinéo-soudanienne } \\
\text { (ou péri-forestière) du plateau } \\
\text { méridional }\end{array}$ & $\begin{array}{l}\text { Sols ferralitiques orthiques remaniés, } \\
\text { orthiques rajeunis et appauvris. }\end{array}$ \\
\hline \multirow[t]{3}{*}{$\begin{array}{l}\text { Forêt tropicale } \\
\text { dense humide }\end{array}$} & $\begin{array}{l}\text { Forêt dense humide } \\
\text { sempervirente } \\
\text { atlantique }\end{array}$ & Sols ferralitiques jaunes topomorphes. \\
\hline & $\begin{array}{l}\text { Forêt toujours } \\
\text { verte congolaise } \\
\text { du Dja }\end{array}$ & $\begin{array}{l}\text { Sols ferralitiques rouges orthiques, } \\
\text { nodaux, jaunes topomorphes remaniés, } \\
\text { associations ferralitiques jaunes remaniés, } \\
\text { hydromorphes indifférenciés. }\end{array}$ \\
\hline & $\begin{array}{l}\text { Forêt semi-caducifoliée } \\
\text { (ou semi-décidue) }\end{array}$ & $\begin{array}{l}\text { Sols ferrallitiques rouges orthiques, } \\
\text { nodaux, ferralitiques jaunes remaniés à } \\
\text { l'est. }\end{array}$ \\
\hline \multirow[t]{3}{*}{ Montagne } & Forêt submontagnarde & $\begin{array}{l}\text { Association de sols ferralitiques, } \\
\text { orthiques peu profonds et pénévolués } \\
\text { lithosoliques et régosoliques des } \\
\text { collines. }\end{array}$ \\
\hline & Forêt montagnarde & $\begin{array}{l}\text { Associations de sols ferralitiques, } \\
\text { humifères et pénévolués; sols } \\
\text { régosoliques et lithosoliques; } \\
\text { andosols et sols bruns eutrophes } \\
\text { d'altitude. }\end{array}$ \\
\hline & Prairie d'altitude & $\begin{array}{l}\text { Associations de sols ferralitiques, } \\
\text { pénévolués; sols régosoliques et } \\
\text { lithosoliques des pentes fortes. }\end{array}$ \\
\hline
\end{tabular}


Boisements des Monts Mandara
Sols minéraux bruts et peu évolués, régosoliques et lithosoliques à faciès ferrugineux et lessivés des montagnes et collines sur roches cristallines.

\begin{tabular}{lll}
\hline Marin et côtier & Mangrove & Sols hydromorphes, sur alluvions marins. \\
\hline Tous & Formations saxicoles & $\begin{array}{l}\text { Cuirasses latéritiques, roches } \\
\text { affleurantes ou immergées. }\end{array}$ \\
\hline
\end{tabular}

Carte des habitats naturels aquatiques par bassin hydrographique du Cameroun

Altitudes en metres

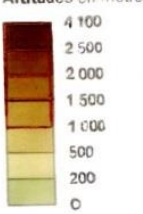

--- Zone inondable

A Sommet

$\approx$ Limite des bassins versants:

$A:$ Atlantique : / $b$ : péninsule de Bakassi : /cr: Cross River (Nigeria) : lew : estuaire du Wouri : /ng : Nyong : /nm : Ntem :

C : Congo : LT : Lac Tchad: $N$ : Niger

O : Ogoué (Gabon) : S : Sanaga

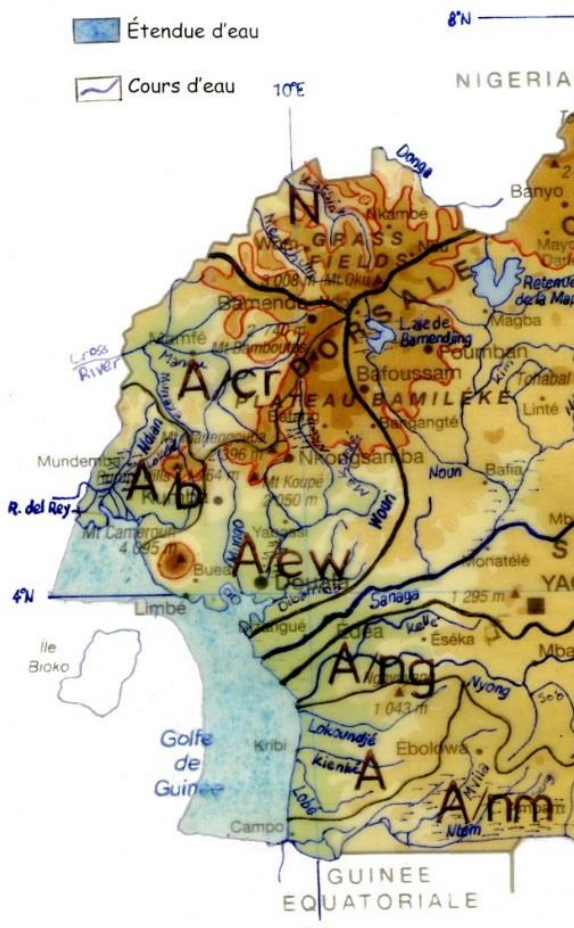

$10^{\circ} \mathrm{E}$

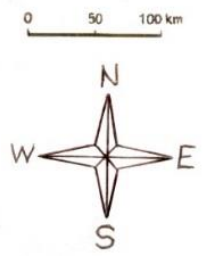

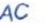

AC

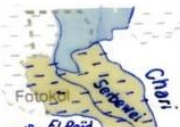

$14^{\circ} \mathrm{E}$ B Beid
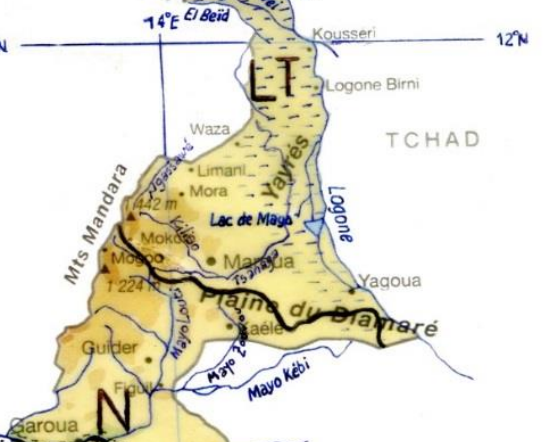


\section{DISCUSSION}

La répartition des habitats terrestres en mosaïques est la conséquence de l'impact des changements climatiques et anthropiques du passé sur les écosystèmes et des dynamiques ayant résulté durant l'histoire paléoenvironnementale avec une répercussion sur la structure, la composition floristique et le paysage des habitats (Maley, 2001; Neumann et al., 2012; Neumann, 2018; Onana, 2019). A cause des limites qui ne sont pas nettes et des incertitudes liées à la cartographie de la biodiversité, les cartes des habitats naturels sont plus un aperçu qu'une carte systématique (Gros-desormeaux, 2015). D'ailleurs étant donné que plusieurs cartes de végétation ont été proposés par différents auteurs tel que Achoundong (2006) ou du couvert végétal (Cameroon Ministry of Forestry and Fauna/ World Resources Institute, 2007), les unités cartographiques seraient légèrement différentes si les délimitations avaient été fabriquées à partir des fonds de carte de végétation de ces dernières. Alors que le paramètre environnemental pour la répartition écosystèmes est le climat, le caractère déterminant pour la distribution spatiale habitats terrestres semble être la nature du sol lorsqu'on se réfère à la carte des sols (Muller et Gavaud, 1979). En effet le sol est une composante majeure de l'habitat car il agit comme un accumulateur, un transformateur et un milieu de transfert pour les cycles biogéochimiques de l'eau, du carbone, des sels minéraux et des métaux. Le microclimat d'un habitat est aussi influencé par le sol en ce que les sols minéraux des habitats ouverts des écosystèmes arides, semi-arides et de savane tropicale boisée, en reflétant la chaleur des rayons du soleil rendent plus chaud le milieu par rapport aux sols des habitats fermés de forêt plus riches en matière organique qui eux absorbent les rayons solaires et maintiennent ainsi une température moins élevée dans le sous-bois (Laban et al., 2018). Quant aux habitats aquatiques, pour chaque cours d'eau, l'habitat est susceptible de changer tous les kilomètres (IUCN, 2010): il y aurait alors par exemple près de 900 sites dans l'habitat du lit de la Sanaga. Chaque site qui se distinguent des autres à son niveau par le débit du fleuve, la profondeur, le ph, l'état biologique (eutrophisation), voire celui de la pollution (Amiet, 1987).

Les résultats obtenus dans ce travail concernent d'abord les communautés végétales. En effet la structure et la composition floristique de la végétation caractérise l'habitat des autres communautés d'êtres vivants. C'est pourquoi ils peuvent être exploités aussi pour les organismes animaux dont la répartition géographique est souvent considérée par rapport aux phytochories (Chirio et LeBreton, 2007; Amiet, 2012; Vivien et Depierre, 2012). Cependant la grande variété d'organismes animaux, leur occupation des milieux naturels à l'échelle des micro-habitats et leurs déplacements souvent saisonniers (migrations) dans diverses localités rendent incertaines la délimitation des habitats des animaux. Ainsi ont été développés beaucoup plus en écologie animale (que végétale) les concepts de biotope (et ainsi de biocénose par rapport à un biotope), micro-habitat, niche écologique, refuge, pour décrire et caractériser les habitats d'organismes animaux suivant la biologie de l'espèce, et par rapport à des taxons parfois du même groupe, les notions de parapatrie, péripatrie, allopatrie, sympatrie ou de vicariance.

\section{Conclusion}

$\mathrm{Ce}$ travail avait pour objectif d'identifier et de cartographier les habitats naturels du Cameroun. Les résultats obtenus donnent la nomenclature ainsi que trois cartes qui montrent la répartition géographique des habitats terrestres et aquatiques. Les habitats terrestres sont répartis selon les types de sol tandis que les habitats aquatiques le sont par bassin versant. Il complète la nomenclature et la cartographie des écosystèmes du Cameroun, enrichit la panoplie d'outils cartographiques sur les paramètres environnementaux biophysiques (climat, 
hydrographie, géologie, géomorphologie, relief, sol, végétation), ou thématiques d'activités humaines (agriculture, pêche, transport, foresterie, ou exploitation minière) du Cameroun. C'est aussi un instrument pour la priorisation des activités de conservation ou d'utilisation durable de la biodiversité, car il permettra de prendre en compte les habitatsclés d'organismes prioritaires pour la conservation, si tant est que parfois, ils sont de superficie réduite par rapport aux standards des superficies minimales des «habitats cruciaux» (MINEPDED, 2014) adoptées par l'administration pour la conservation forestière ou des zones humides. De même, c'est un outil de planification pour les administrations territoriales décentralisées, avec pour perspective, la réalisation de cartes de sites d'intérêt particulier dans leur territoire en vue de promouvoir des activités compatibles avec le développement durable local dont l'écotourisme.

\section{CONFLIT D'INTÉRÊTS}

Les auteurs déclarent n'avoir aucun conflit d'intérêts.

\section{CONTRIBUTIONS DES AUTEURS}

J-MO a initié le projet d'article, assuré la collecte et l'analyse des données, l'interprétation des résultats, et la coordination de la rédaction du manuscrit; JLF a participé à l'analyse des données et a contribué à la rédaction du manuscrit; EHB a contribué à l'interprétation des résultats et à la rédaction du manuscrit; ENT a mis en forme les cartes; et MMAM a participé à l'interprétation des résultats et la rédaction du manuscrit.

\section{REMERCIEMENTS}

Les auteurs expriment leurs remerciements à Monsieur Hervé Chevillotte de l'Institut de Recherche pour le Développement (France) pour son assistance au cours de la préparation et de la rédaction du premier manuscrit, de même qu'aux relecteurs anonymes dont les observations et commentaires ont permis d'améliorer significativement la qualité du premier manuscrit.

\section{RÉFÉRENCES}

Achoundong. 2006. Le Cameroun. Végétation. In Atlas du Cameroun, Collection Atlas de l'Afrique, Ben Yamed D, Houstin N, Seignobos C (eds). Les Editions J.A. : Paris ; 64-65

Amiet J-L. 2012. Les Rainettes du Cameroun (Amphibiens Anoures). Jean-Louis Amiet et la Nef des livres (eds). SaintNazaire.

Bachman S, Moat J. 2012. GeoCAT - an open source tool for rapid Red List assessments. BGjournal, 9 (1): 11-13.

Bachman S, Moat J, Hill AW, de la Torre J, Scott B. 2011. Supporting Red List threat assessments with GeoCAT: geospatial conservation assessment tool. ZooKeys, 150: 117-126. DOI: 10.3897/zookeys.150.2109

Beentje H. 2010. The Kew Plant Glossary. Kew publishing. Royal Botanic Gardens, Kew; 55.

Cameroon Ministry of Forestry and Fauna/ World Resources Institute 2007. Vegetation cover in Cameroon. Interactive Atlas of forestry resources of Cameroon, version 2.0.

Charahabil MM, César B, Hamadou B, Ndiaye S, Diatta M. 2018. Diversité et structure des espaces végétalisés urbains de la ville de Ziguinchor, Sénégal. Int. J. Biol. Chem. Sci., 12(4): 1650-1666. DOI: https://dx.doi.org/10.4314/ijbcs.v12i4.12

Chirio L, LeBreton M. 2007. Atlas des reptiles du Cameroun. Publications Scientifiques du Muséum 67. IRD Editions; 10-19.

Darbyshire I, Anderson S, Asatryan A, Byfield A, Cheek M, Clubbe C, Ghrabi Z, Harris T, Heatubun CD, Kalema J, Magassouba S, McCarthy B, Milliken W, de Montmollin B, Lughadha EN, Onana JM, Saïdou D, Sârbu A, Shrestha K, Radford EA. 2017. Important Plant Areas: revised selection criteria for a 
global approach to plant conservation. Biodivers Conserv., 26(8): 1767-1800. DOI: 10.1007/s10531-017-1336-6.

Folahan SON, Dissou EF, Akouehou GS, Tente BAH, Boko M. 2018. Ecologie et structure des groupements végétaux des écosystèmes de la Lama au Sud-Bénin. Int. J. Biol. Chem. Sci., 12(1): 322-340. DOI:

https://dx.doi.org/10.4314/ijbcs.v12i1.26

Garba A, Djima IT, Abdou L, Mahamane A. 2017. Caractérisation de la végétation ligneuse du bassin versant de la Maggia dans la commune rurale de Bagaroua (région de Tahoua). Int. J. Biol. Chem. Sci., 11(2): 571-584. DOI: https://dx.doi.org/10.4314/ijbcs.v11i2.4

Gillson L, Ladle JR, Araùjo MB. 2011. Baselines, patterns and process. In Conservation Biogeography, Ladle RJ, Whittaker RJ (eds). Willey-Blackwell; 30-44.

Gros-Désormeaux J-R. 2015. Les incertitudes dans la cartographie de la biodiversité : le cas des Znieff dans les Antilles françaises. Nat. Sc. Soc., 23: 256-265. DOI: $10.1051 / \mathrm{nss} / 2015053$.

IUCN Standards and Petitions Subcommittee. 2010. Guidelines for Using the IUCN Red List Categories and Criteria. Version 8.1. Prepared by the Standards and Petitions Subcommittee in March 2010. Downloadable from:

http://intranet.iucn.org/webfiles/doc/SSC /RedList/RedListGuidelines.pdf.

file:///C:/Users/Onana/Downloads/175_

RedListGuidelines.pdf.

IUCN 2014a. SIS version 2.0 Manual - July 2014 - Plants. http://sis.iucnsis.org

IUCN 2014b. SIS version 2.0 Manual- July 2014 - Marine. http://sis.iucnsis.org

IUCN 2016 A Global Standard for the Identification of Key Biodiversity Areas, Version 1.0. First edition. Gland, Switzerland: IUCN (International Union For Conservation of Nature), Cambridge, UK. www.iucn.org.
IUCN 2018a. Threats Classification Scheme Version 3.2. http://www.iucnredlist.org/ technical-documents/classificationschemes/threats-classification-scheme

IUCN 2018b. Classification Scheme, version 3.1. http://www.iucnredlist.org/technicaldocuments/classificationschemes/habitats-classification-schemever3.

Le Breton TD, Zimmer HC, Gallagher RV, Cox M, Allen S, Auld TD. 2019. Using IUCN criteria to perform rapid assessments of at-risk taxa. Biodivers Conserv., $\quad$ 28(4): 863-883. https://doi.org/10.1007/s10531-01901697-9

Letouzey R. 1985. Carte Phytogéographique du Cameroun au 1:500.000. Accompagnée de la Notice de la carte phytogéographique du Cameroun au 1:500 000. Institut de la Carte Internationale de la Végétation. Toulouse, France.

Limoges B, Boisseau G, Gratton L, Kasisi R. 2013. Terminologie relative à la conservation de la biodiversité in situ. Le Naturaliste Canadien, 137(2): 21-27.

Maley J. 2001. La destruction catastrophique des forêts d'Afrique centrale survenue il y a environ 2500 ans exerce encore une influence majeure sur la répartition actuelle des formations végétales. Syst. Geogr. Pl., 71: 777-796.

MINEPDED. 2014. 20+ Objectifs de biodiversité pour le Cameroun. Priorités de la stratégie et plan d'action national pour la biodiversité II. In Stratégie et Plan d'Action National pour la Biodiversité - Version II, 2012, République du Cameroun (ed.) MINEPDED; 7-11.

Morin S. 1979. Relief et hydrographie. In Atlas de la République Unie $d u$ Cameroun, Laclavère G (ed.) Les Editions J.A. Paris; 5-8.

Muller J-P, Gavaud M. 1979. Les sols. In Atlas de la République Unie $d u$ 
Cameroun, Laclavère G. (ed.). Les Editions J.A.: Paris; 25-27.

Neumann K. 2018. Development of Plant Food Production in the West African Savannas: Archaeobotanical Perspectives. Oxford Research Encyclopedia, African History. Online Publication Oxford University Press USA, $2019 . \quad$ DOI: 10.1093/acrefore/9780190277734.013.13 8

Neumann K, Bostoen K, Höhn A, Kahlheber S, Ngomanda A, Tchiengué B. 2012. First farmers in the Central African rainforest: A view from southern Cameroon. Quat. Int. 249: 53-42. DOI:10.1016/j.quaint.2011.03.024

Nguéguim JR, Zapfack L, Onana JM, Noiha NV, Onana D, Betti JL, Gachet S, Riera B. 2016. Can the Mangombe forest plantation contribute to restore and conserve biodiversity? Int. J. Biol. Chem. Sci., 10(1): 355-368. DOI: http://dx.doi.org/10.4314/ijbcs.v10i1.27

Onana JM. 2018. Cartographie des écosystèmes du Cameroun. Int. J. Biol. Chem. Sci., 12(2): 940-957. DOI: https://dx.doi.org/10.4314/ijbcs.v12i2.25

Onana JM. 2019. Ecosystèmes du Cameroun. Tendances $d u$ Passé, Paysages Modernes. Éditions Universitaires Européennes: Mauritius.

Onana JM, Cheek M. 2011. Red Data Book of the Flowering Plants of Cameroon: IUCN Global Assessments. Royal Botanic Gardens, Kew and IRADNational Herbarium of Cameroon. Kew Publishing. London.

Onana JM, Mbome MJ, Mekembom YN. 2017. The North-South Synergy: The National Herbarium and Limbe Botanic Garden experience. Sci. Dan. Series B, Biologica, 6: 117-139.

Ouattara D, Kouame D, Tiebre M-S, Kouadio YJ-C, N'guessan KE. 2016. Biodiversité végétale et valeur d'usage en zone soudanienne de la Côte d'Ivoire. Int. J. Biol. Chem. Sci., 10(3): 1122-1138. DOI: http://dx.doi.org/10.4314/ijbcs.v10i3.18
République du Cameroun. 2012. Stratégie et Plan d'Action National pour la Biodiversité - Version II 2012 MINEPDED ; 1-189.

République du Cameroun 2014. Cinquième Rapport National du Cameroun à la Convention de la Diversité Biologique. MINEPDED; 27. www.cbd.int

Ridle BR, Ladle RJ, Lourie SA, Whittaker RJ. 2011. Basic biogeography: estimating biodiversity and mapping nature. In Conservation Biogeography, Ladle RJ, Whittaker RJ (eds). Willey-Blackwell: London; 47-92.

Sambieni KR, Bilosso MA, Toyi MS, Sambieni E, Natta AK, Occhiuto R, Bogaert J. 2018. La biodiversité des parcelles habitées en zone périurbaine à Kinshasa: déterminants sociobiophysiques et représentations. Int. J. Biol. Chem. Sci., 12(3): 1164-1183. DOI: https://dx.doi.org/10.4314/ijbcs.v12i3.8

Secrétariat de la Convention sur la Biodiversité. 2002. Stratégie Mondiale pour la Conservation des Plantes. Botanic Garden Conservation International : Londres; 3-15. www.bgci.org.uk

Secrétariat de la Convention sur la Diversité Biologique. 2004. Approche Par Écosystème (Lignes Directrices de la CDB) Montréal: Secrétariat de la Convention sur la diversité biologique; 6.

Sadik S. 2013. Ecology: meaning and definitions. In Environmental Geography.

http://shadmansadik.blogspot.com/2013/ 11/ecology-meaning-anddefinitions.html

Schatz GE, Lowry II PP, Onana JM, Stévart T, Deblauwe V. 2019. Diospyros crassiflora. In The IUCN Red List of Threatened Species 2019: e.T33048A2831968. http://dx.doi.org/10.2305/IUCN.UK.201 9-1.RLTS.T33048A2831968.en

Tchatchou B, Sonwa DJ, Ifo S, Tiani AM. 2015. Déforestation et dégradation des 
forêts dans le Bassin du Congo. État des lieux, causes actuelles et perspectives. CIFOR, Papier occasionnel 120. Bogor, Indonésie; 3-5.

Tchawa P. 2006. Le Cameroun. Relief et hydrographie. In Atlas du Cameroun, Collection Atlas de l'Afrique, Ben Yamed D, Houstin N, Seignobos C. (eds). Les Editions J.A.: Paris; 58-59.

Thiam A, Samba SAN, Noba K, Ndiaye JP, Diatta M, Wade M. 2015. Etude de la variation de la végétation en milieux salé et acide au Sénégal. Int. J. Biol. Chem. Sci., 9(1): 155-175. DOI: http://dx.doi.org/10.4314/ijbcs.v9i1.15

Tiokeng B, Mapongmetsem P-M, Nguetsop VF, Tacham WN. 2015. Biodiversité floristique et régénération naturelle sur les Hautes Terres de Lebialem (Ouest Cameroun). Int. J. Biol. Chem. Sci., 9(1): 56-68.

DOI:

http://dx.doi.org/10.4314/ijbcs.v9i1.6
UDEV 2013. Définition des termes habitat, biotope et niche écologique. http://www. supagro.fr/ress-pepites/Opale/Processus Ecologiques/co/Ha_Definitions.html.

UICN 2012a. Catégories et Critères de l'UICN pour la Liste Rouge: Version 3.1. Deuxième édition. Gland, Suisse et Cambridge, Royaume Uni.

Vivien J, Depierre D. 2012. Mammifères Sauvages du Cameroun. Ed. Nguila Kérou : Saint Berthevin, France.

Willis F, Moat J, Paton A. 2003. Defining a role for herbarium data in Red List assessments: a case study of Plectranthus from eastern and southern tropical Africa. Biodivers. Conserv., 12: 1513-1552.

Witté I, Touroult J. 2014 Répartition de la biodiversité en France métropolitaine : une synthèse des Atlas faunistiques, VertigO - la revue électronique en sciences de l'environnement, 14(1). DOI: 10.4000/vertigo.14645. 